\title{
A New Spectral-Homotopy Perturbation Method and Its Application to Jeffery-Hamel Nanofluid Flow with High Magnetic Field
}

\begin{abstract}
Ahmed A. Khidir
Faculty of Technology of Mathematical Sciences and Statistics, Alneelain University, Algamhoria Street, P.O. Box 12702, Khartoum, Sudan

Correspondence should be addressed to Ahmed A. Khidir; ahmed.khidir@yahoo.com

Received 23 May 2013; Revised 20 November 2013; Accepted 4 December 2013

Academic Editor: Ali Cemal Benim

Copyright (C) 2013 Ahmed A. Khidir. This is an open access article distributed under the Creative Commons Attribution License, which permits unrestricted use, distribution, and reproduction in any medium, provided the original work is properly cited.

We present a new modification of the homotopy perturbation method (HPM) for solving nonlinear boundary value problems. The technique is based on the standard homotopy perturbation method, and blending of the Chebyshev pseudospectral methods. The implementation of the new approach is demonstrated by solving the Jeffery-Hamel flow considering the effects of magnetic field and nanoparticle. Comparisons are made between the proposed technique, the standard homotopy perturbation method, and the numerical solutions to demonstrate the applicability, validity, and high accuracy of the present approach. The results demonstrate that the new modification is more efficient and converges faster than the standard homotopy perturbation method.
\end{abstract}

\section{Introduction}

Many problems in the fields of physics, engineering, and biology are modeled by coupled linear or nonlinear systems of partial or ordinary differential equations. Compared to nonlinear equations, linear equations can be easily solved and finding analytical solutions to nonlinear problems on finite or infinite domains is one of the most challenging problems. Such problems do not usually admit closed from analytic solutions and in most cases we look for finding approximate solutions using numerical approximation techniques. Nonnumerical approaches include the classical power-series method and its variants for systems of nonlinear differential equations with small or large embedded parameters. One of these methods is the homotopy perturbation method (HPM). This method, which is a combination of homotopy in topology and classic perturbation techniques, provides us with a convenient way to obtain analytic or approximate solutions for a wide variety of problems arising in different scientific fields by continuously deforming the difficult problem into a set of simple linear problems that are easy to solve. It was proposed first by $\mathrm{He}[1-4]$. $\mathrm{He}$ has successfully used the method to solve many types of linear and nonlinear differential equations such as Lighthill equation [1], Duffing equation [2], Blasius equation [5], wave equations [4], and boundary value problems [6]. Homotopy perturbation method has been recently intensively studied by many authors and they used the method for solving nonlinear problems and some modifications of this method have been published [7-12] to facilitate, make accurate calculations, and accelerate the rapid convergence of the series solution and reduce the size of work. Jalaal et al. [13] used HPM to investigate the acceleration motion of a vertically falling spherical particle in incompressible Newtonian media. Jalaal and Ganji [14] applied HPM successfully on the problem of unsteady rolling motion of spheres in inclined tubes filled with incompressible Newtonian fluids. They found that the inclination angle does not affect the acceleration duration.

The application of the homotopy perturbation method in linear and non-linear models has been developed by many scientists and engineers, because this method provides us with a convenient way to obtain analytic or approximate solutions for a wide variety of problems arising in different scientific fields by continuously deforming the difficult problem into a set of simple linear problems that are easy to solve (e.g., see $[12,15,16])$. 
The homotopy perturbation method has been applied on the MHD Jeffery-Hamel problem by [17]. This model is one of the most applicable cases of the incompressible viscous fluid flow through convergent-divergent channels in fluid mechanics, civil, environmental, mechanical, and biomechanical engineering. Jeffery [18] and Hamel [19] were the first persons who discussed this case, and so it is known as Jeffery-Hamel problem. They presented an exact similarity solution of the Navier-Stokes equations in the special case of two-dimensional flow through a channel with inclined plane walls meeting at a vertex and with a source or sink at the vertex. The problem has been well extensively studied by several authors and discussed in many articles and textbooks. The study of [20] considers the steady two-dimensional radial flow of viscous fluid between plane walls which either converge or diverge. In the Ph.D. thesis [21], we find that JefferyHamel flow is used as asymptotic boundary conditions to examine steady two-dimensional flow of a viscous fluid in a channel. But, here, certain symmetric solutions of the flow have been considered, although asymmetric solutions are both possible and of physical interest [22]. The classical Jeffery-Hamel problem was extended in [23] to include the effects of an external magnetic field on an electrically conducting fluid. Recent studies to solving the Jeffery-Hamel flow problem include perturbation techniques [24], Adomian decomposition method [25-27], homotopy analysis method [28-30], optimal homotopy asymptotic method [31], and variational iteration method [17].

In this work, we present an alternative and improved form of the HPM called spectral-homotopy perturbation method (SHPM) that blends the traditional homotopy perturbation method with the Chebyshev spectral collocation method. The advantage of this approach is that it is more flexible than HPM for choosing a linear operator and initial guess. In HPM, one is restricted to choosing a linear operator and initial approximation that would make the integration of the higher-order differential equations possible whereas the SHPM allows us to have a wider range of selecting linear operators and an initial guess may be used as long as it satisfies the boundary conditions.

We have applied the new technique to find an approximate solution of MHD Jeffery-Hamel flow and to study the effect of nanoparticle volume fraction on the velocity profile. We have made a comparison between the current results and the numerical solutions in plots and tables to show the applicability, validity, and accuracy of this method. The obtained results give rapid convergence, good accuracy and suggest that this newly improvement technique introduces power for solving nonlinear boundary value problems and several advantages of the SHPM over the HPM approach are also pointed out.

\section{Mathematical Formulation}

We consider the steady two-dimensional flow of an incompressible conducting viscous fluid from a source or sink at the intersection between two rigid plane walls that the angel between them is $2 \alpha$ as shown in Figure 1. The grid walls

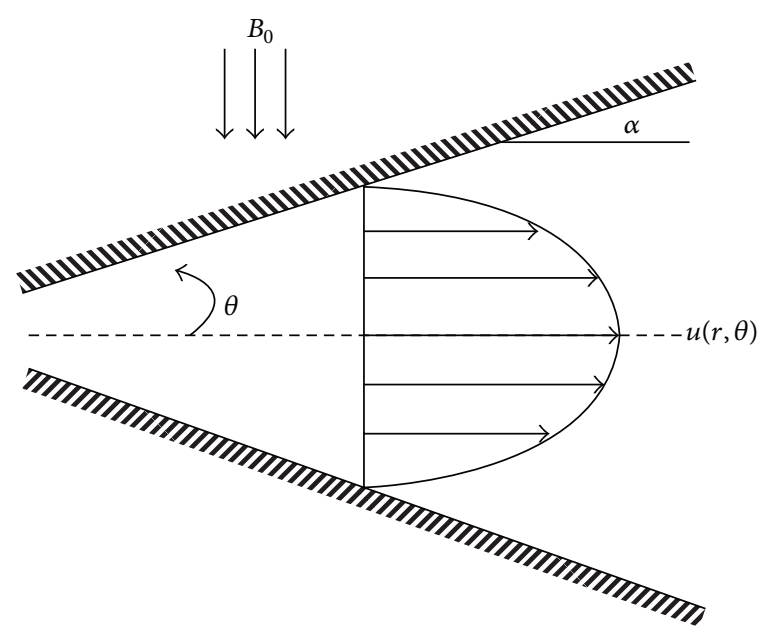

FIgURE 1: Geometry of the MHD Jeffery-Hamel flow.

are considered to be divergent if $\alpha>0$ and convergent if $\alpha<0$. We assume that the velocity is only along radial direction and depends on $r$ and $\theta$ so that $\mathbf{v}=(u(r, \theta), 0)$ only and, further, we assume that there is no magnetic field in the $z$-direction. The velocity is assumed only along radial direction and depends on $r$ and $\theta$. Conservation of mass and momentum for two-dimensional flow in the cylindrical coordinate can be expressed as follows:

$$
\begin{gathered}
\frac{\rho_{\mathrm{nf}}}{r} \frac{\partial}{\partial r}(r u(r, \theta))=0 \\
u(r, \theta) \frac{\partial u(r, \theta)}{\partial r}=-\frac{1}{\rho_{\mathrm{nf}}} \frac{\partial P}{\partial r}+v_{\mathrm{nf}} \\
\times\left[\frac{\partial^{2} u(r, \theta)}{\partial r^{2}}+\frac{1}{r} \frac{\partial u(r, \theta)}{\partial r}\right. \\
\left.+\frac{1}{r^{2}} \frac{\partial^{2} u(r, \theta)}{\partial \theta^{2}}-\frac{u(r, \theta)}{r^{2}}\right] \\
-\frac{\sigma B_{0}^{2}}{\rho_{\mathrm{nf}} r^{2}} u(r, \theta) \\
\frac{1}{\rho_{\mathrm{nf}} r} \frac{\partial P}{\partial \theta}-\frac{2 v_{\mathrm{nf}}}{r^{2}} \frac{\partial u(r, \theta)}{\partial \theta}=0
\end{gathered}
$$

where $P$ is the fluid pressure, $B_{0}$ is the electromagnetic induction, and $\sigma$ is the conductivity of the fluid.

The effective density $\rho_{\mathrm{nf}}$, the effective dynamic viscosity $\mu_{\mathrm{nf}}$, and the kinematic viscosity $v_{\mathrm{nf}}$ of the nanofluid are given as $[33,34]$

$$
\begin{gathered}
\rho_{\mathrm{nf}}=\rho_{f}(1-\phi)+\rho_{s} \phi, \\
\mu_{\mathrm{nf}}=\frac{\mu_{f}}{(1-\phi)^{2.5}}, \\
v_{\mathrm{nf}}=\frac{\mu_{\mathrm{nf}}}{\rho_{\mathrm{nf}}}
\end{gathered}
$$


Here, $\phi$ is the solid volume fraction, $\mu_{f}$ is the viscosity of the basic fluid, and $\rho_{f}$ and $\rho_{s}$ are the densities of the pure fluid and nanoparticle, respectively. The continuity equation (1) implies that

$$
u(r, \theta)=\frac{f(\theta)}{r}
$$

Using the following dimensionless parameters [28]:

$$
F(\eta)=\frac{f(\theta)}{f_{\max }}, \quad \eta=\frac{\theta}{\alpha}
$$

and eliminating $P$ from (2) and (3), we obtain the following third-order non-linear ordinary differential equation for the normalized function profile $F(\eta)$ :

$$
\begin{aligned}
F^{\prime \prime \prime}(\eta) & +2 \alpha \operatorname{Re}\left[(1-\phi)^{2.5}\left(1-\phi+\frac{\rho_{s}}{\rho_{f}} \phi\right)\right] \\
& \times F^{\prime}(\eta) F(\eta)+\alpha^{2}\left(4-(1-\phi)^{2.5} \mathrm{Ha}\right) F^{\prime}(\eta)=0,
\end{aligned}
$$

subject to the boundary conditions

$$
F(0)=1, \quad F^{\prime}(0)=0, \quad F(1)=0,
$$

where Re is the Reynolds number given by

$$
\begin{aligned}
\operatorname{Re} & =\frac{\alpha f_{\max }}{v_{f}}=\frac{U_{\max } r \alpha}{v_{f}} \\
& =\left(\begin{array}{c}
\text { divergent chanel: } \alpha>0, f_{\max }>0 \\
\text { convergent chanel: } \alpha<0, f_{\max }<0
\end{array}\right),
\end{aligned}
$$

where $U_{\max }$ is the velocity at the center of the channel $(r=0)$ and $\mathrm{Ha}=\sqrt{\sigma B_{0}^{2} / \rho_{f} v_{f}}$ is the Hartmann number.

\section{Homotopy Perturbation Method}

To illustrate the basic ideas of the homotopy perturbation method, we consider the following nonlinear differential equation:

$$
A(u)-f(\mathbf{r})=0, \quad \mathbf{r} \in \Omega
$$

with the boundary conditions

$$
B\left(u, \frac{\partial u}{\partial n}\right)=0, \quad \mathbf{r} \in \Gamma,
$$

where $A$ is a general operator, $B$ is a boundary operator, $f(\mathbf{r})$ is a known analytic function, and $\Gamma$ is the boundary of the domain $\Omega$. The operator $A$ can, generally speaking, be divided into two parts, $L$ and $N$, where $L$ is linear and $N$ is nonlinear. Equation (10) can therefore be written as follows:

$$
L(u)+N(u)-f(\mathbf{r})=0 .
$$

By the homotopy technique $[29,30]$, we construct a homotopy $v(r, p): \Omega \times[0,1] \rightarrow \mathfrak{R}$ which satisfies

$$
\begin{array}{r}
H(v, p)=(1-p)\left[L(v)-L\left(u_{0}\right)\right]+p[A(v)-f(\mathbf{r})]=0, \\
p \in[0,1], \mathbf{r} \in \Omega
\end{array}
$$

or

$$
H(v, p)=L(v)-L\left(u_{0}\right)+p L\left(u_{0}\right)+p[N(v)-f(\mathbf{r})]=0,
$$

where $p \in[0,1]$ is an embedding parameter and $u_{0}$ is an initial approximation of (10), which satisfies the boundary conditions. Obviously, from (13), we have

$$
\begin{aligned}
& H(v, 0)=L(v)-L\left(u_{0}\right)=0, \\
& H(v, 1)=A(v)-f(\mathbf{f} r)=0 .
\end{aligned}
$$

We can assume that the solution of (10) can be written as a power series in $p$, as follows:

$$
v=v_{0}+p v_{1}+p^{2} v_{2}+\cdots .
$$

Setting $p=1$ results in the approximation to the solution of (10)

$$
u=\lim _{p \rightarrow 1} v=v_{0}+v_{1}+v_{2}+\cdots
$$

\section{Spectral-Homotopy Perturbation Method}

The basic idea of SHPM for solving a non-linear differential equation is summarized as follows:

(1) linearize the non-linear differential equation by applying HPM,

(2) rewrite the resulting linear differential equation in a system of linear equations using Chebyshev pseudospectral collocation method,

(3) solve the system of equations to get the solution of origin differential equation.

In this section, we describe the new technique of SHPM for solving the governing MHD Jeffery-Hamel problem. The method is based on Chebyshev pseudospectral collocation methods and on the HPM described in the previous section. In applying the SHPM, we begin by transforming the physical region $[0,1]$ into the region $[-1,1]$ on which the Chebyshev spectral method can be applied by using the transformations

$$
\eta=\frac{1+\xi}{2}, \quad \xi \in[-1,1]
$$

It is also convenient to make the boundary conditions homogeneous by using the transformation

$$
F(\eta)=f(\xi)+1-\eta^{2} .
$$


Substituting (18) and (19) in the governing equation (7) gives

$$
\begin{aligned}
& 8 f^{\prime \prime \prime}(\xi)+a_{1}(\eta) f^{\prime}(\xi)+a_{2}(\eta) f \\
& \quad+a_{3}(\eta) f^{\prime}(\xi) f(\xi)=\Phi(\eta) .
\end{aligned}
$$

Equation (20) is solved subject to the boundary conditions

$$
f(-1)=0, \quad f(1)=0, \quad f^{\prime}(-1)=0,
$$

where

$$
\begin{aligned}
a_{1}(\eta)= & 8 \alpha^{2}+4 \alpha \operatorname{Re}(1-\phi)^{2.5}\left(1-\eta^{2}\right) \\
& \times\left(1+\frac{\rho_{s}}{\rho_{f}} \phi-2 \alpha^{2} \mathrm{Ha}-\phi\right) \\
a_{2}(\eta)= & -4 \eta \alpha \operatorname{Re}(1-\phi)^{2.5}\left(1-\phi+\frac{\rho_{s}}{\rho_{f}} \phi\right), \\
a_{3}= & 4 \alpha \operatorname{Re}(1-\phi)^{2.5}\left(1-\phi+\frac{\rho_{s}}{\rho_{f}} \phi\right), \\
\Phi(\eta)= & 2 \eta \alpha^{2}\left(4-(1-\phi)^{2.5} \mathrm{Ha}\right) \\
& +4 \alpha \operatorname{Re}(1-\phi)^{2.5}\left(\eta-\eta^{3}\right)\left(1-\phi+\frac{\rho_{s}}{\rho_{f}} \phi\right) .
\end{aligned}
$$

The initial approximation for the solution of (20) is taken to be the solution of the nonhomogeneous linear part of (20) which is given by

$$
8 f_{0}^{\prime \prime \prime}(\xi)+a_{1}(\eta) f_{0}^{\prime}(\xi)+a_{2}(\eta) f_{0}=\Phi(\eta),
$$

subject to the boundary conditions

$$
f_{0}(-1)=0, \quad f_{0}(1)=0, \quad f_{0}^{\prime}(-1)=0 .
$$

The solution of (23) and (24) can be obtained by applying the Chebyshev pseudospectral method (see $[35,36]$ for details). The unknown function $f_{0}(\xi)$ is approximated as a truncated series of Chebyshev polynomials of the form

$$
f_{0}(\xi) \approx f_{0}^{N}(\xi)=\sum_{k=0}^{N} f_{k} T_{k}\left(\xi_{j}\right), \quad j=0,1, \ldots, N
$$

where $T_{k}$ is the $k$ th Chebyshev polynomial, $f_{k}$ are coefficients, and $\xi_{0}, \xi_{1}, \ldots, \xi_{N}$ are Gauss-Lobatto collocation points defined by [35]

$$
\xi_{j}=\cos \frac{\pi j}{N}, \quad j=0,1, \cdots, N
$$

The derivatives of the function $f_{0}(\xi)$ at the collocation points are represented as

$$
\frac{d^{r} f_{0}(\xi)}{d \xi^{r}}=\sum_{k=0}^{N} \mathbf{D}_{k j}^{r} f_{0}\left(\xi_{j}\right)
$$

where $r$ is the order of differentiation and $\mathbf{D}=2 \mathscr{D}$ with $\mathscr{D}$ being the Chebyshev spectral differentiation matrix whose entries are defined as (see, e.g., [35])

$$
\begin{aligned}
& \mathscr{D}_{j k}=\frac{c_{j}}{c_{k}} \frac{(-1)^{j+k}}{\xi_{j}-\xi_{k}}, \quad j \neq k ; j, k=0,1, \ldots, N, \\
& \mathscr{D}_{k k}=-\frac{\xi_{k}}{2\left(1-\xi_{k}^{2}\right)} \quad k=1,2, \ldots, N-1, \\
& \mathscr{D}_{00}=\frac{2 N^{2}+1}{6}=-\mathscr{D}_{N N} .
\end{aligned}
$$

Substituting (25)-(27) in (23) and (24) yields

$$
\mathbf{A} f_{0}=\Phi
$$

subject to the boundary conditions

$$
f_{0}\left(\xi_{N}\right)=0, \quad f_{0}\left(\xi_{0}\right)=0, \quad \sum_{k=0}^{N} \mathscr{D}_{N k} f_{0}\left(\xi_{k}\right)=0
$$

where

$$
\begin{gathered}
\mathbf{A}=8 \mathscr{D}^{3}+a_{1} \mathscr{D}+a_{2}, \\
f_{0}=\left[f_{0}\left(\xi_{0}\right), f_{0}\left(\xi_{1}\right), \ldots, f_{0}\left(\xi_{N}\right)\right]^{T}, \\
\Phi=\left[\Phi\left(\eta_{0}\right), \Phi\left(\eta_{1}\right), \ldots, \Phi\left(\eta_{N}\right)\right]^{T}, \\
a_{j}=\operatorname{diag}\left[a_{j}\left(\eta_{0}\right), a_{j}\left(\eta_{1}\right), \ldots, a_{j}\left(\eta_{N}\right)\right]^{T}, \quad j=1,2,
\end{gathered}
$$

where diag[] is a diagonal matrix of size $N \times N$ and $T$ denotes the transpose. The matrix A has dimensions $N \times N$ while matrix $\Phi$ has dimensions $N \times 1$. To incorporate the boundary conditions (24) to (29), we delete the first and the last rows and columns of $\mathbf{A}$ and delete the first and last elements of $f_{0}$ and $\Phi$. The condition $f_{0}^{\prime}(-1)=0$ is incorporated on the resulting of the modified matrix $\mathbf{A}$ by replacing last row with the last row of Chebyshev spectral differentiation matrix and setting the resulting last element of the modified matrix $\Phi$ to be zero as shown in the following equation:

$$
\begin{gathered}
\left(\begin{array}{ccccc}
A_{0,0} & A_{0,1} & \cdots & A_{0, N-1} & A_{0, N} \\
A_{1,0} & & & A_{1, N} \\
\vdots & & \mathbf{A} & \vdots \\
A_{N-2,0} & & & A_{N-2, N} \\
\mathscr{D}_{N, 0} & \mathscr{D}_{N, 1} & \cdots & \mathscr{D}_{N, N-1} & \mathscr{D}_{N, N} \\
A_{N, 0} & A_{N, 1} & \cdots & A_{N, N-1} & A_{N, N}
\end{array}\right) \\
\quad \times\left(\begin{array}{c}
f\left(\xi_{0}\right) \\
f\left(\xi_{1}\right) \\
\vdots \\
f\left(\xi_{N-2}\right) \\
f\left(\xi_{N-1}\right) \\
f\left(\xi_{N}\right)
\end{array}\right)=\left(\begin{array}{c}
0 \\
\Phi\left(\eta_{1}\right) \\
\vdots \\
\Phi\left(\eta_{N-2}\right) \\
0 \\
0
\end{array}\right)
\end{gathered}
$$

Thus, the solution $f_{0}$ is determined from the equation

$$
f_{0}=\widetilde{\mathbf{A}}^{-1} \widetilde{\boldsymbol{\Phi}},
$$


where $\widetilde{\mathbf{A}}$ and $\widetilde{\Phi}$ are the modified matrices of $\mathbf{A}$ and $\Phi$, respectively. The solution (33) provides us with the initial approximation for the SHPM solution of (20). The higher approximations are obtained by constructing a homotopy for the government equation (20) as follows:

$$
\begin{aligned}
\mathscr{H}(\mathbf{F} ; p)= & \mathscr{L}(\mathbf{F})-\mathscr{L}\left(\mathbf{F}_{0}\right)+p L(\mathbf{F}) \\
& +p\left[a_{3}(\eta) \mathbf{F}^{\prime} \mathbf{F}-\Phi\right]=0,
\end{aligned}
$$

where $\mathscr{L}$ is a linear operator which is taken to be

$$
\mathscr{L}=8 \frac{d^{3}}{d \xi^{3}}+a_{1} \frac{d}{d \xi}+a_{2}
$$

and $\mathbf{F}$ is an approximate series solution of (20) given by

$$
\mathbf{F}=f_{0}+p f_{1}+p^{2} f_{2}+\cdots+p^{m} f_{m}=\sum_{i=0}^{m} p^{i} f_{i} .
$$

Substituting (35) and (36) in (34) gives

$$
\begin{aligned}
8 \frac{d^{3} \mathbf{F}}{d \xi^{3}} & +a_{1} \frac{d \mathbf{F}}{d \xi}+a_{2} \mathbf{F}-8 \frac{d^{3} f_{0}}{d \xi^{3}} \\
& -a_{1} \frac{d f_{0}}{d \xi}-a_{2} f_{0}+p\left[8 \frac{d^{3} \mathbf{F}}{d \xi^{3}}+a_{1} \frac{d \mathbf{F}}{d \xi}+a_{2} \mathbf{F}\right] \\
& +p\left[a_{3}(\eta) \mathbf{F}^{\prime} \mathbf{F}-\Phi\right]=0 .
\end{aligned}
$$

To get more higher-order approximations for (20), we compare between the coefficients of $p^{i}$ of both sides in (37). We have

$$
\begin{gathered}
p^{1}: \mathscr{L} f_{1}+\mathscr{L} f_{0}+a_{3}\left(f_{0}^{\prime} f_{0}-\Phi\right)=0, \\
p^{2}: \mathscr{L} f_{2}+a_{3}\left(f_{0}^{\prime} f_{1}+f_{1}^{\prime} f_{0}\right)=0, \\
\vdots \\
p^{i}: \mathscr{L} f_{i}+(1-\chi) \mathscr{L} f_{0}-a_{3}(1-\chi) \Phi \\
+a_{3} \sum_{n=0}^{i-1} f_{n}^{\prime} f_{i-1-n}=0, \quad i=1,2, \ldots,
\end{gathered}
$$

where $\chi=0$ if $i=1$ and $\chi=1$ if $i>1$. Thus, the $i$ th-order approximation is given by the following system of matrices:

$$
\mathbf{A} f_{i}=\mathbf{B}_{i},
$$

subject to the boundary conditions

$$
\begin{gathered}
f_{i}\left(\xi_{0}\right)=0, \quad f_{i}\left(\xi_{N}\right)=0, \quad \sum_{k=0}^{N} \mathscr{D}_{N k} f_{i}\left(\xi_{k}\right)=0, \\
\mathbf{B}_{i}=a_{3}(1-\chi) \Phi-(1-\chi) \mathbf{A} f_{0}-a_{3} \sum_{n=0}^{i-1} f_{n}^{\prime} f_{i-1-n}
\end{gathered}
$$

where $f_{i}=\left[f_{i}\left(\xi_{0}\right), f_{i}\left(\xi_{1}\right), \ldots, f_{i}\left(\xi_{N}\right)\right]^{T}$ and $a_{3}=\operatorname{diag}\left[a_{3}\left(\eta_{0}\right)\right.$, $\left.a_{3}\left(\eta_{1}\right), \ldots, a_{3}\left(\eta_{N}\right)\right]^{T}$. To incorporate the boundary conditions
(40) to (39), we delete the first and the last rows and columns of $\mathbf{A}$ and delete the first and last elements of $f_{i}$ and $\mathbf{B}_{i}$. The boundary condition $f_{i}^{\prime}(-1)=0$ is incorporated on the resulting of the modified matrix $\mathbf{A}$ by replacing last row by the last row of Chebyshev spectral differentiation matrix and setting the resulting last element of the modified matrix $\mathbf{B}_{i}$ to be zero. Finally, the solution $f_{i}$ is determined from the following equation:

$$
f_{i}=\widetilde{\mathbf{A}}^{-1} \widetilde{\mathbf{B}}_{i},
$$

where $\widetilde{\mathbf{B}}_{i}$ is the modified matrix of $\mathbf{B}_{i}$. Thus, starting from the initial approximation $f_{0}$, higher-order approximations $f_{m}$, for $m \geq 1$, can be obtained through the recursive formula (42). The solution (42) provides us with the highest order approximation for the SHPM solution of the governing equation (20). The series $f_{i}$ is convergent for most cases. However, the convergence rate depends on the nonlinear operator of (20). The following opinions are suggested and proved by $\mathrm{He}[1,2]$ :

(i) the second derivative of $N(v)$ with respect to $v$ must be small because the parameter $p$ may be relatively large; that is, $p \rightarrow 1$;

(ii) the norm of $L^{-1}(\partial N / \partial v)$ must be smaller than one so that the series converges.

This is the same strategy that is used in the SHPM approach.

Here, we observe that the main difference between HPM and SHPM is that the solutions are obtained by solving a system of higher-order ordinary differential equations in the HPM while for the SHPM solutions are obtained by solving a system of linear algebraic equations of the form (42) that are easier to solve.

\section{Results and Discussion}

In this section, we present the results obtained using the SHPM and the numerical solution for MHD Jeffery-Hame, flow with nanoparticle. Here, we used the inbuilt MATLAB boundary value problem solver bvp4c for the numerical solution approach. In generating the presented results, it was determined through numerical experimentation that $N=80$ and we considered the fourth-order of SHPM which gave sufficient accuracy for the method. In this study, copper $(\mathrm{Cu})$ is considered as nanoparticles with water being as the base fluid and we assumed that the base fluid and the nanoparticles are in thermal equilibrium and no slip occurs between them. The density of water is $\rho_{f}=997.1$ and the density of $\mathrm{Cu}$ is $\rho_{s}=8933$.

Figures 2 and 3 show, firstly, the influence of the various physical parameters on the velocity profiles and, secondly, a comparison between the present results and numerical results to give a sense of the accuracy and convergence rate of the SHPM. The figures show that there is very good match between the two sets of results even at very low orders of SHPM approximations series compared with the numerical results. These findings firmly establish SHPM as an accurate alternative to HPM. Table 1 gives a comparison of SHPM 


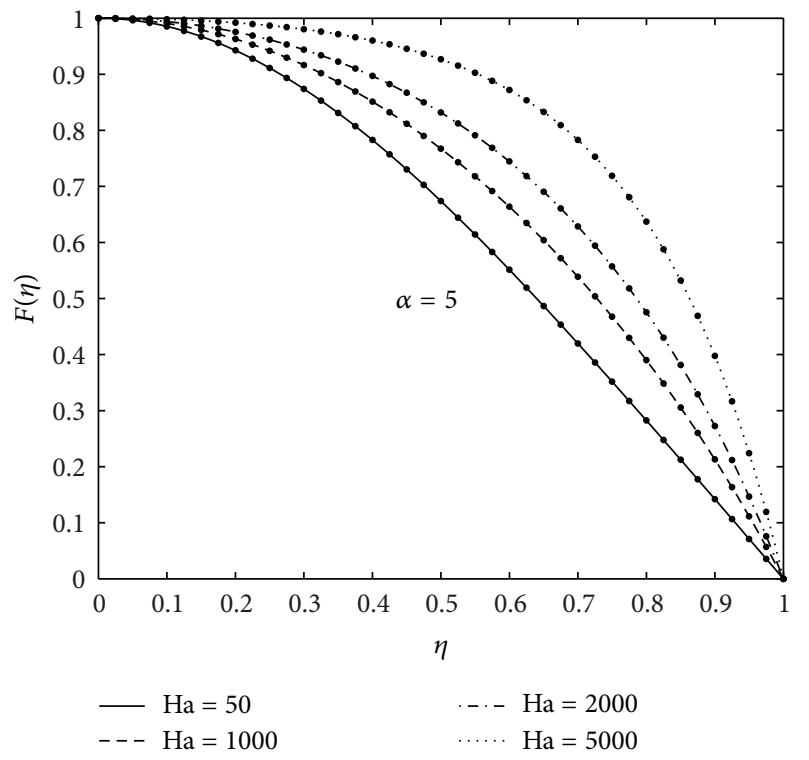

(a)

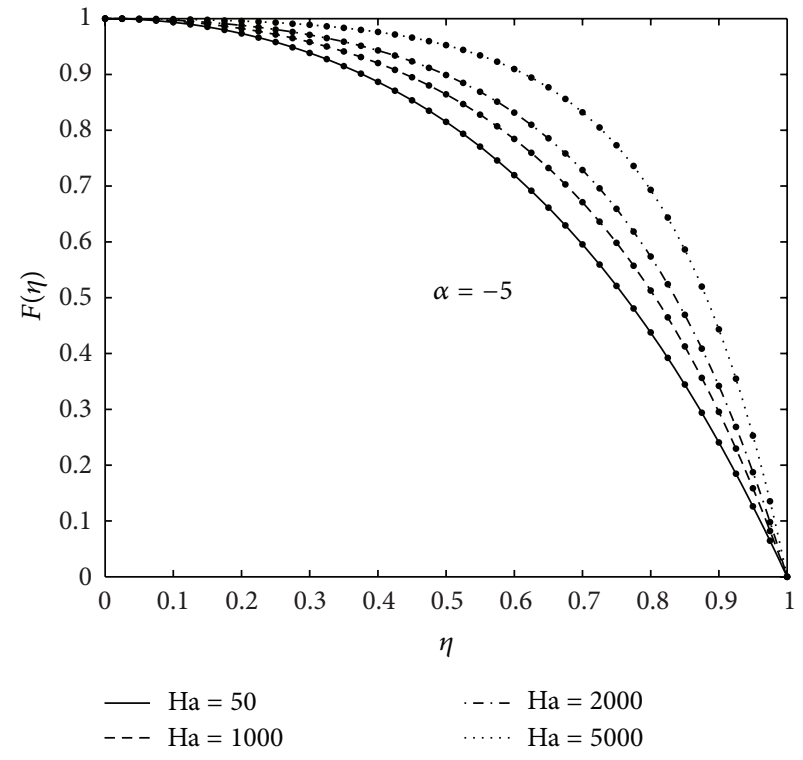

(b)

FIGURE 2: Comparison of the numerical results (filled circles) and SHPM approximation for the velocity profile when Re $=50, \phi=0.1$ and different values of Ha.

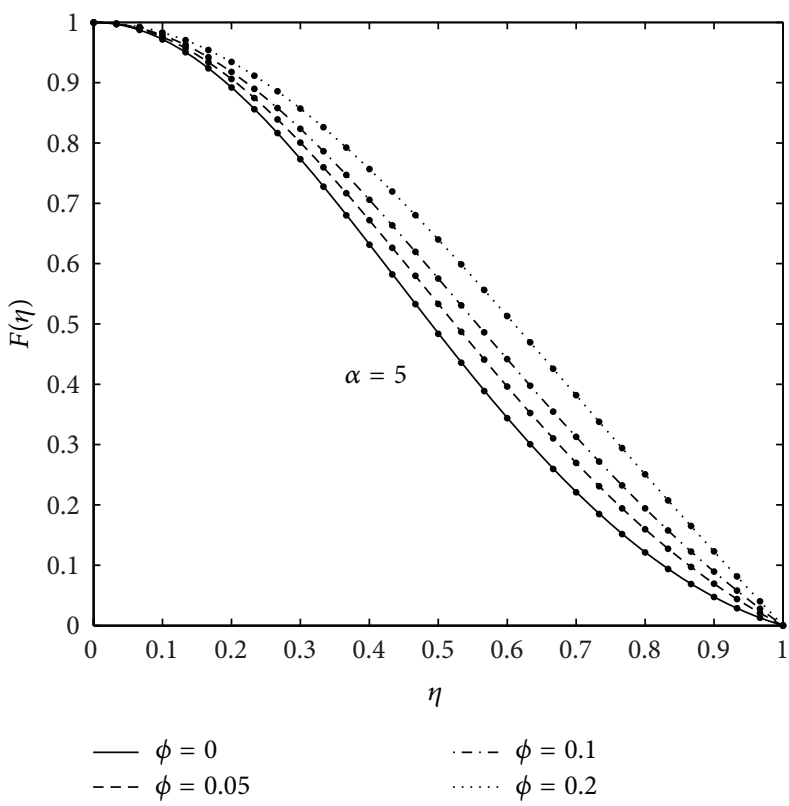

(a)

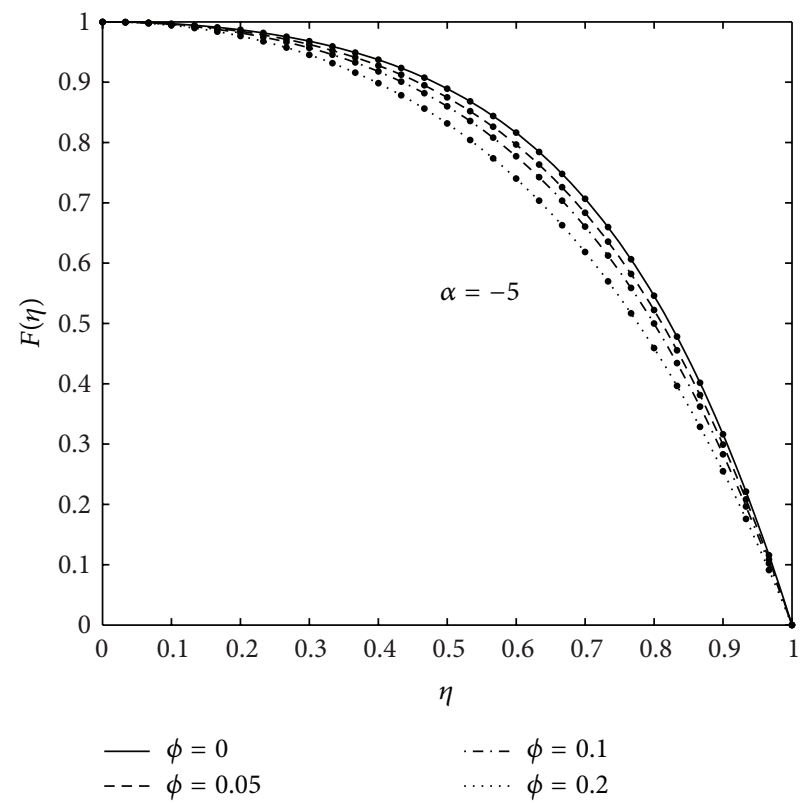

(b)

FIGURE 3: Comparison of the numerical results (filled circles) and SHPM approximation for the velocity profile when $\mathrm{Re}=130, \mathrm{Ha}=50$ and different values of $\phi$ for $\mathrm{Cu}$-water.

results at 2 nd and 3 rd orders of approximation against the numerical results for fixed values of $\mathrm{Re}=10, \mathrm{Ha}=200$, and $\alpha=5$ when $\eta$ and $\phi$ are varied. It can be seen, from Table 1 , that SHPM results converge rapidly to the numerical solution and similar results achieved between the numerical results and 3rd order of SHPM approximation up to 8 decimal places. Table 2 shows a comparison of HPM and SHPM results at different orders of approximation against the numerical results at selected values of magnetic field and nanoparticle volume fraction for fixed values of $\operatorname{Re}$ and $\alpha$. Convergence of the SHPM is achieved at the 2 nd order of approximation up to 8 decimal places as observed in Table 2 even for large values of Ha. In Table 3, we made a comparison between the numerical results, HPM, 10th-order homotopy analysis method [32], and variational iteration method [17] and different order of SHPM of the fluid velocity $F(\eta)$ and the second derivative 
TABLE 1: Comparison of the numerical results against the SHPM approximate solutions for $F(\eta)$ and $F^{\prime \prime}(\eta)$ when $\operatorname{Re}=10$, Ha $=200$, and $\alpha=5$.

\begin{tabular}{|c|c|c|c|c|c|c|c|}
\hline & \multirow{2}{*}{$\eta$} & \multicolumn{3}{|c|}{$F(\eta)$} & \multicolumn{3}{|c|}{$F^{\prime \prime}(\eta)$} \\
\hline & & 2 nd order & 3 rd order & Numerical & 2 nd order & 3 rd order & Numerical \\
\hline \multirow{11}{*}{$\phi=0.1$} & 0.0 & 1 & 1 & 1 & -1.97361423 & -1.97361423 & -1.97361423 \\
\hline & 0.1 & 0.99013256 & 0.99013258 & 0.99013258 & -1.97286367 & -1.97286367 & -1.97286367 \\
\hline & 0.2 & 0.96053716 & 0.96053725 & 0.96053725 & -1.97132614 & -1.97132614 & -1.97132614 \\
\hline & 0.3 & 0.91122738 & 0.91122758 & 0.91122758 & -1.97114172 & -1.97114171 & -1.97114172 \\
\hline & 0.4 & 0.84220211 & 0.84220246 & 0.84220246 & -1.97587434 & -1.97587434 & -1.97587434 \\
\hline & 0.5 & 0.75340988 & 0.75341039 & 0.75341039 & -1.99052431 & -1.99052431 & -1.99052431 \\
\hline & 0.6 & 0.64469883 & 0.64469947 & 0.64469947 & -2.02157959 & -2.02157959 & -2.02157959 \\
\hline & 0.7 & 0.51575172 & 0.51575242 & 0.51575242 & -2.07714031 & -2.07714032 & -2.07714032 \\
\hline & 0.8 & 0.36600474 & 0.36600533 & 0.36600533 & -2.16716308 & -2.16716309 & -2.16716309 \\
\hline & 0.9 & 0.19454749 & 0.19454782 & 0.19454782 & -2.30388760 & -2.30388760 & -2.30388760 \\
\hline & 1.0 & 0 & 0 & 0 & -2.50253230 & -2.50253230 & -2.50253230 \\
\hline \multirow{11}{*}{$\phi=0.4$} & 0.0 & 1 & 1 & 1 & -1.97636754 & -1.97636754 & -1.97636754 \\
\hline & 0.1 & 0.99011749 & 0.99011749 & 0.99011749 & -1.97717852 & -1.97717852 & -1.97717852 \\
\hline & 0.2 & 0.96046170 & 0.96046171 & 0.96046171 & -1.97979610 & -1.97979610 & -1.97979610 \\
\hline & 0.3 & 0.91100600 & 0.91100602 & 0.91100602 & -1.98477509 & -1.98477509 & -1.98477509 \\
\hline & 0.4 & 0.84169981 & 0.84169985 & 0.84169985 & -1.99304375 & -1.99304375 & -1.99304375 \\
\hline & 0.5 & 0.75245937 & 0.75245942 & 0.75245942 & -2.00591025 & -2.00591025 & -2.00591025 \\
\hline & 0.6 & 0.64315460 & 0.64315467 & 0.64315467 & -2.02507402 & -2.02507402 & -2.02507403 \\
\hline & 0.7 & 0.51359211 & 0.51359218 & 0.51359218 & -2.05264442 & -2.05264442 & -2.05264442 \\
\hline & 0.8 & 0.36349409 & 0.36349415 & 0.36349415 & -2.09116967 & -2.09116967 & -2.09116968 \\
\hline & 0.9 & 0.19247275 & 0.19247278 & 0.19247278 & -2.14368045 & -2.14368045 & -2.14368046 \\
\hline & 1.0 & 0 & 0 & 0 & -2.21375299 & -2.21375298 & -2.21375298 \\
\hline
\end{tabular}

TABLE 2: Comparison of the numerical results against the SHPM approximate solutions for $F^{\prime \prime}(0)$ when $\operatorname{Re}=10$ and $\alpha=5$.

\begin{tabular}{|c|c|c|c|c|c|c|c|}
\hline \multirow{2}{*}{$\phi$} & \multirow{2}{*}{$\mathrm{Ha}$} & \multicolumn{4}{|c|}{ SHPM } & \multirow{2}{*}{ HPM } & \multirow{2}{*}{ Numerical } \\
\hline & & 1 st order & 2 nd order & 3rd order & 4 th order & & \\
\hline \multirow{4}{*}{0} & 0 & -2.25205783 & -2.25205817 & -2.25205817 & -2.25205817 & -2.2522802 & -2.25205817 \\
\hline & 50 & -2.18134736 & -2.18134748 & -2.18134748 & -2.18134748 & -2.1795097 & -2.18134748 \\
\hline & 100 & -2.11325918 & -2.11325920 & -2.11325920 & -2.11325920 & -2.1107698 & -2.11325920 \\
\hline & 500 & -1.65047007 & -1.65046878 & -1.65046877 & -1.65046877 & -1.6734400 & -1.65046877 \\
\hline \multirow{4}{*}{0.05} & 0 & -2.21083280 & -2.21083294 & -2.21083294 & -2.21083294 & -2.2110158 & -2.21083294 \\
\hline & 50 & -2.14969149 & -2.14969153 & -2.14969153 & -2.14969153 & -2.1484672 & -2.14969153 \\
\hline & 100 & -2.09055197 & -2.09055197 & -2.09055197 & -2.09055197 & -2.0889107 & -2.09055197 \\
\hline & 500 & -1.68097975 & -1.68097904 & -1.68097904 & -1.68097904 & -1.6988408 & -1.68097904 \\
\hline \multirow{4}{*}{0.1} & 0 & -2.17511715 & -2.17511720 & -2.17511720 & -2.17511720 & -2.1752670 & -2.17511720 \\
\hline & 50 & -2.12250597 & -2.12250599 & -2.12250599 & -2.12250599 & -2.1217211 & -2.12250599 \\
\hline & 100 & -2.07140445 & -2.07140446 & -2.07140446 & -2.07140446 & -2.0703633 & -2.07140446 \\
\hline & 500 & -1.71124187 & -1.71124151 & -1.71124151 & -1.71124151 & -1.7246699 & -1.71124151 \\
\hline
\end{tabular}

$F^{\prime \prime}(\eta)$. The convergence of the SHPM is achieved at the 4 th order whereas convergence is not achieved even at the 10th order of HAM approximation. Clearly, SHPM converges at least four times as fast and demonstrates the higher accuracy than HAM, HPM, and VIM. For this reason, SHPM has been used to investigate the effects of physical parameters of the problem. The effect of the magnetic field on the velocity profile is displayed in Figures 2(a) and 2(b) of convergent and divergent channels for fixed values of nanoparticle volume fraction and fixed Reynolds number. The figures indicate that the increase in the Hartmann number leads to increase in the velocity profiles for both convergent and divergent channels which has a great effect on the performance of the system. This is due to the fact that the variation of Ha leads to the variation of the Lorentz force due to the magnetic field, and the Lorentz force produces more resistance to the transport phenomena. Similar result has been reported by [26, 27, 32]. Figure 3 shows the effect of nanoparticle volume fraction on 
TABLE 3: Comparison of the numerical results against the SHPM, HPM, HAM, and VIM approximate solutions for $F(\eta)$ and $F^{\prime \prime}(\eta)$ when $\operatorname{Re}=50, \alpha=5, \mathrm{Ha}=0$, and $\phi=0$.

\begin{tabular}{|c|c|c|c|c|c|c|c|c|}
\hline & \multirow{2}{*}{$\eta$} & \multirow{2}{*}{ HPM } & \multirow{2}{*}{ Reference [32] } & \multirow{2}{*}{ Reference [17] } & \multicolumn{3}{|c|}{ SHPM } & \multirow{2}{*}{ Numerical } \\
\hline & & & & & 2nd order & 3 rd order & 4 th order & \\
\hline \multirow{5}{*}{$F(\eta)$} & 0 & 1 & 1 & 1 & 1 & 1 & 1 & 1 \\
\hline & 0.25 & 0.894960 & 0.894242 & 0.894243 & 0.894238 & 0.894242 & 0.894242 & 0.894242 \\
\hline & 0.50 & 0.627220 & 0.626948 & 0.626953 & 0.626937 & 0.626948 & 0.626948 & 0.626948 \\
\hline & 0.75 & 0.302001 & 0.301991 & 0.301998 & 0.301982 & 0.301990 & 0.301990 & 0.301990 \\
\hline & 1 & 0 & 0 & 0 & 0 & 0 & 0 & 0 \\
\hline \multirow{5}{*}{$F^{\prime \prime}(\eta)$} & 0 & -3.539214 & -3.539417 & -3.539369 & -3.539546 & -3.539416 & -3.539416 & -3.539416 \\
\hline & 0.25 & -2.661930 & -2.662083 & -2.662048 & -2.662153 & -2.662084 & -2.662084 & -2.662084 \\
\hline & 0.50 & -0.879711 & -0.879791 & -0.879791 & -0.879612 & -0.879793 & -0.879794 & -0.879794 \\
\hline & 0.75 & -0.447331 & 0.447242 & 0.447052 & 0.447350 & 0.447244 & 0.447244 & 0.447244 \\
\hline & 1 & -0.854544 & 0.854367 & 0.854440 & 0.854239 & 0.854369 & 0.854369 & 0.854369 \\
\hline
\end{tabular}

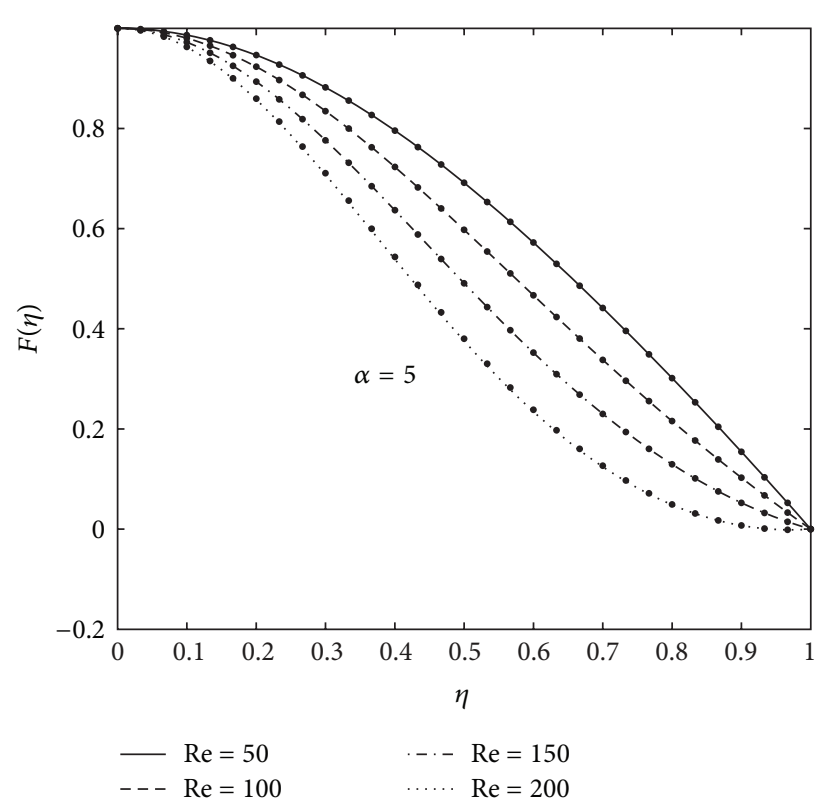

(a)

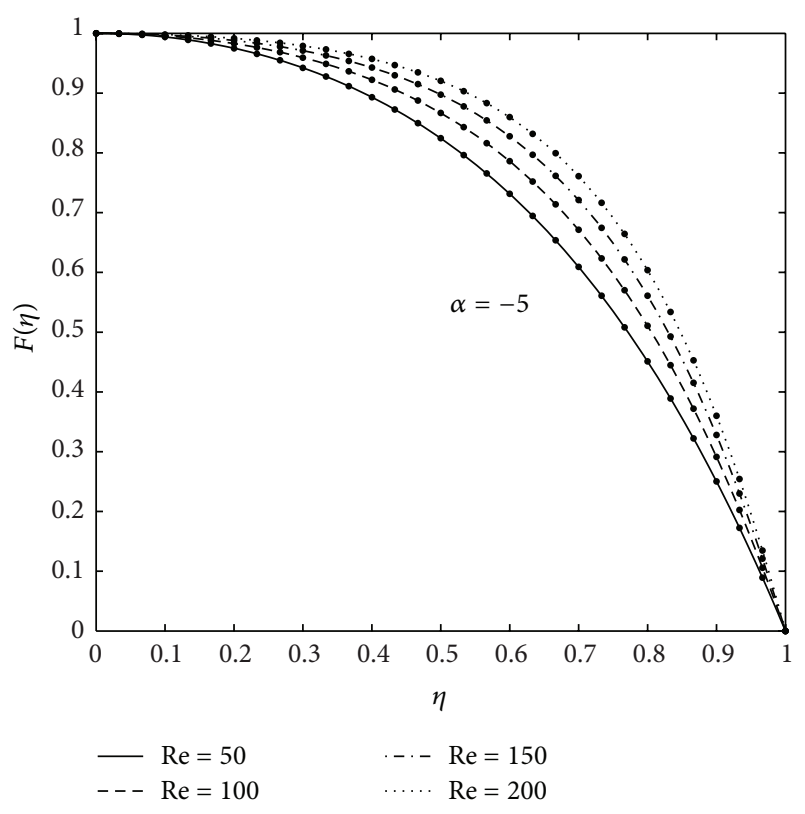

(b)

FIGURE 4: Comparison of the numerical results (filled circles) and SHPM approximation for the velocity profile when Ha $=200, \phi=0.1$ and different values of Re.

the fluid velocity for fixed Hartmann and Reynolds numbers. It is observed that the fluid velocity increases with the increase in the value of $\phi$ in the case of diverging channels, and backflow phenomenon is expected for small values of $\phi$ and large values of $\mathrm{Re}$, while the fluid velocity decreases as the nanoparticle volume fraction increases for the converging channels case. Figure 4 illustrates the effect of increasing Reynolds numbers on the fluid velocity. It was found that, from Figures 4(a) and 4(b) for inflow system, back flow is prevented in the case of convergent channels but is possible for large Reynolds numbers in the case of divergent channels; that is, there is a reverse condition for outflow regime (see $[17,32])$.

\section{Conclusion}

In this work, we have proposed a modification of the standard homotopy perturbation method for solving nonlinear ordinary differential equations. The method has been used to solve the 3rd-order MHD Jeffery-Hamel flow with nanoparticle. Tables and graphical results are presented to show the accuracy and the convergence rate of the SHPM and to investigate the effects of different physical parameters on the flow as well.

The main conclusions emerging from this study are as follows. 
(1) SHPM is highly accurate, efficient and converges rapidly with a few iterations required to achieve the accuracy of the numerical results compared with the standard HPM. For example, in this study, it was found that only fourth iteration of SHPM was sufficient to give good agreement with the numerical results.

(2) The method proposes a standard way of choosing the linear operators and initial approximations by using any form of initial guess as long as it satisfies the boundary conditions while the initial guess in the HPM can be selected that will make the integration of the higher-order deformation equations possible.

(3) The SHPM converges much faster than HPM. For example, in the study above, it was found that the third-order SHPM approximation was sufficient to give good agreement with the numerical results.

(4) The increase of volume fraction causes an increase in the fluid velocity profile for of diverging channels while the velocity decreases for the converging channels case.

(5) The fluid velocity increases with increasing Hartman numbers for both diverging and converging channels cases.

Finally, the spectral-homotopy perturbation method described above has high accuracy and is simple for nonlinear boundary value problems compared with the standard homotopy perturbation method. Because of its efficiency and ease of use, the SHPM can also be used to solve nonlinear BVPs in place of the traditional Runge-Kutta methods, finite differences, and Keller-box.

\section{References}

[1] J. H. He, "Homotopy perturbation technique," Computer Methods in Applied Mechanics and Engineering, vol. 178, no. 3-4, pp. 257-262, 1999.

[2] J. H. He, "Homotopy perturbation method: a new nonlinear analytical technique," Applied Mathematics and Computation, vol. 135, no. 1, pp. 73-79, 2003.

[3] J. H. He, "Comparison of homotopy perturbation method and homotopy analysis method," Applied Mathematics and Computation, vol. 156, no. 2, pp. 527-539, 2004.

[4] J. H. He, "Application of homotopy perturbation method to nonlinear wave equations," Chaos, Solitons and Fractals, vol. 26, no. 3, pp. 695-700, 2005.

[5] J. H. He, "A simple perturbation approach to Blasius equation," Applied Mathematics and Computation, vol. 140, no. 2-3, pp. 217-222, 2003.

[6] J.H. He, "Homotopy perturbation method for solving boundary value problems," Physics Letters A, vol. 350 , no. 1-2, pp. 87-88, 2006.

[7] Z. M. Odibat, "A new modification of the homotopy perturbation method for linear and nonlinear operators," Applied Mathematics and Computation, vol. 189, no. 1, pp. 746-753, 2007.

[8] A. Beléndez, C. Pascual, M. Ortuño, T. Beléndez, and S. Gallego, "Application of a modified He's homotopy perturbation method to obtain higher-order approximations to a nonlinear oscillator with discontinuities," Nonlinear Analysis: Real World Applications, vol. 10, no. 2, pp. 601-610, 2009.

[9] A. Beléndez, C. Pascual, S. Gallego, M. Ortuño, and C. Neipp, "Application of a modified He's homotopy perturbation method to obtain higher-order approximations of an $x^{1 / 3}$ force nonlinear oscillator," Physics Letters A, vol. 371, no. 5-6, pp. 421-426, 2007.

[10] Z. Odibat and S. Momani, "Modified homotopy perturbation method: application to quadratic Riccati differential equation of fractional order," Chaos, Solitons and Fractals, vol. 36, no. 1, pp. 167-174, 2008.

[11] A. Golbabai and B. Keramati, "Modified homotopy perturbation method for solving Fredholm integral equations," Chaos, Solitons and Fractals, vol. 37, no. 5, pp. 1528-1537, 2008.

[12] A. Belndez, C. Pascual, T. Belendez, and A. Hernandez, "Solution for an anti-symmetric quadratic nonlinear oscillator by a modified He's homotopy perturbation method," Nonlinear Analysis: Real World Applications, vol. 10, no. 1, pp. 416-427, 2009.

[13] M. Jalaal, D. D. Ganji, and G. Ahmadi, "Analytical investigation on acceleration motion of a vertically falling spherical particle in incompressible Newtonian media," Advanced Powder Technology, vol. 21, no. 3, pp. 298-304, 2010.

[14] M. Jalaal and D. D. Ganji, "On unsteady rolling motion of spheres in inclined tubes filled with incompressible Newtonian fluids," Advanced Powder Technology, vol. 22, no. 1, pp. 58-67, 2011.

[15] L. Cveticanin, "Homotopy-perturbation method for pure nonlinear differential equation," Chaos, Solitons and Fractals, vol. 30, no. 5, pp. 1221-1230, 2006.

[16] F. Shakeri and M. Dehghan, "Solution of delay differential equations via a homotopy perturbation method," Mathematical and Computer Modelling, vol. 48, no. 3-4, pp. 486-498, 2008.

[17] Z. Z. Ganji, D. D. Ganji, and M. Esmaeilpour, "Study on nonlinear Jeffery-Hamel flow by He's semi-analytical methods and comparison with numerical results," Computers and Mathematics with Applications, vol. 58, no. 11-12, pp. 2107-2116, 2009.

[18] G. B. Jeffery, "The two-dimensional steady motion of a viscous fluid," Philosophical Magazine, vol. 6, pp. 455-465, 1915.

[19] G. Hamel, "Spiralförmige bewgungen zäher flüssigkeiten," Jahresbericht der Deutschen Mathematiker-Vereinigung, vol. 25, pp. 34-60, 1916.

[20] L. Rosenhead, "The steady two-dimensional radial flow of viscous fluid between two inclined plane walls," Proceedings of the Royal Society A, vol. 175, pp. 436-467, 1940.

[21] M. Reza Sadri, Channel entrance flow [Ph.D. thesis], Department of Mechanical Engineering, The University of Western Ontario, Ontario, Canada, 1997.

[22] I. J. Sobey and P. G. Drazin, "Bifurcations of two-dimensional channel flows," Journal of Fluid Mechanics, vol. 171, pp. 263-287, 1986.

[23] W. I. Axford, "The magnetohydrodynamic Jeffrey-Hamel problem for a weakly conducting fluid," Quarterly Journal of Mechanics and Applied Mathematics, vol. 14, no. 3, pp. 335-351, 1961.

[24] A. McAlpine and P. G. Drazin, "On the spatio-temporal development of small perturbations of Jeffery-Hamel flows," Fluid Dynamics Research, vol. 22, no. 3, pp. 123-138, 1998.

[25] Q. Esmaili, A. Ramiar, E. Alizadeh, and D. D. Ganji, "An approximation of the analytical solution of the Jeffery-Hamel flow by decomposition method," Physics Letters A, vol. 372, no. 19, pp. 3434-3439, 2008. 
[26] O. D. Makinde and P. Y. Mhone, "Hermite-Padé approximation approach to MHD Jeffery-Hamel flows," Applied Mathematics and Computation, vol. 181, no. 2, pp. 966-972, 2006.

[27] O. D. Makinde, "Effect of arbitrary magnetic Reynolds number on MHD flows in convergent-divergent channels," International Journal of Numerical Methods for Heat and Fluid Flow, vol. 18, no. 6, pp. 697-707, 2008.

[28] D. G. Domairry, A. Mohsenzadeh, and M. Famouri, "The application of homotopy analysis method to solve nonlinear differential equation governing Jeffery-Hamel flow," Communications in Nonlinear Science and Numerical Simulation, vol. 14, no. 1, pp. 85-95, 2009.

[29] S. J. Liao, The proposed homotopy analysis technique for the solution of nonlinear problems [Ph.D. thesis], Shanghai Jiao Tong University, Shanghai, China, 1992.

[30] S. J. Liao, Beyond Perturbation: Introduction to Homotopy Analysis Method, Chapman \& Hall/CRC Press, 2003.

[31] M. Esmaeilpour and D. D. Ganji, "Solution of the JefferyHamel flow problem by optimal homotopy asymptotic method," Computers and Mathematics with Applications, vol. 59, no. 11, pp. 3405-3411, 2010.

[32] S. S. Motsa, P. Sibanda, F. G. Awad, and S. Shateyi, "A new spectral-homotopy analysis method for the MHD JefferyHamel problem," Computers and Fluids, vol. 39, no. 7, pp. 12191225, 2010.

[33] K. Khanafer, K. Vafai, and M. Lightstone, "Buoyancy-driven heat transfer enhancement in a two-dimensional enclosure utilizing nanofluids," International Journal of Heat and Mass Transfer, vol. 46, no. 19, pp. 3639-3653, 2003.

[34] F. M. Hady, F. S. Ibrahim, S. M. Abdel-Gaied, and M. R. Eid, "Radiation effect on viscous flow of a nanofluid and heat transfer over a nonlinearly stretching sheet," Nanoscale Research Letters, vol. 7, article 229, 2012.

[35] C. Canuto, M. Y. Hussaini, A. Quarteroni, and T. A. Zang, Spectral Methods in Fluid Dynamics, Springer, Berlin, Germany, 1988.

[36] L. N. Trefethen, Spectral Methods in MATLAB, SIAM, 2000. 

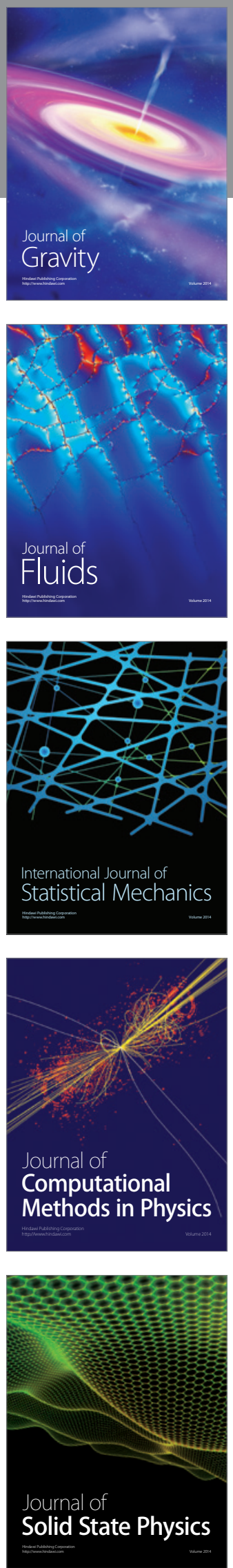

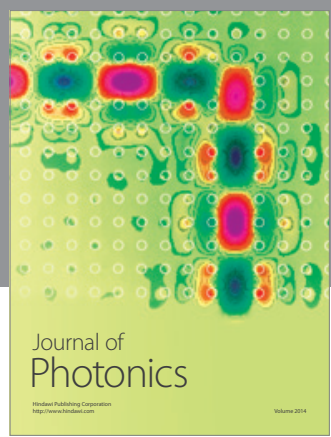

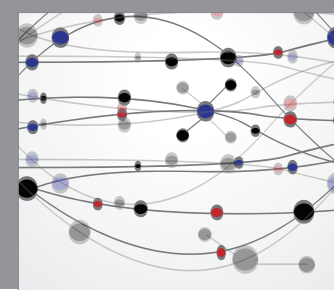

The Scientific World Journal

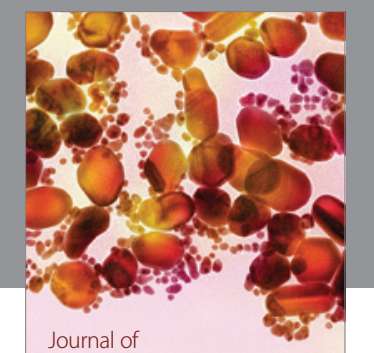

Soft Matter
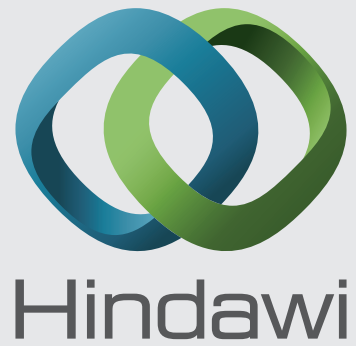

Submit your manuscripts at

http://www.hindawi.com
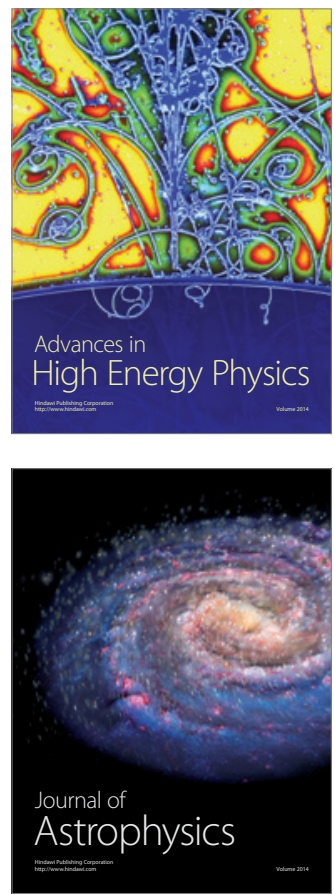
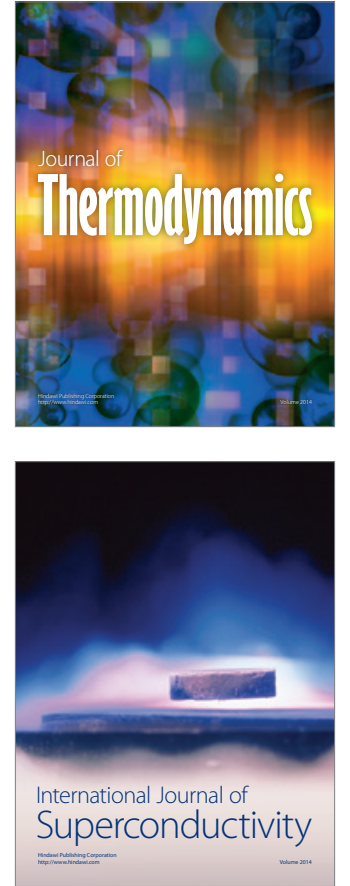
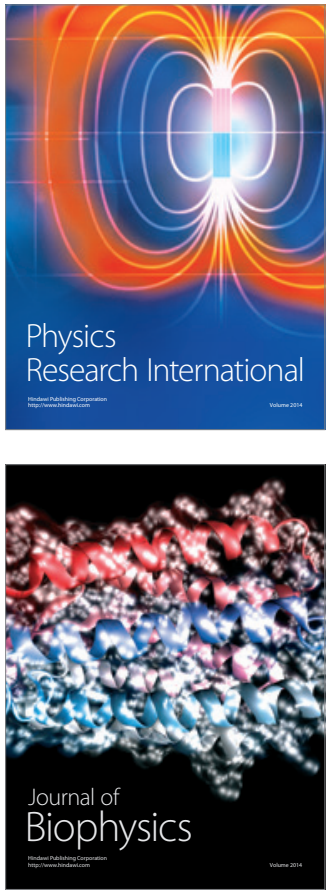
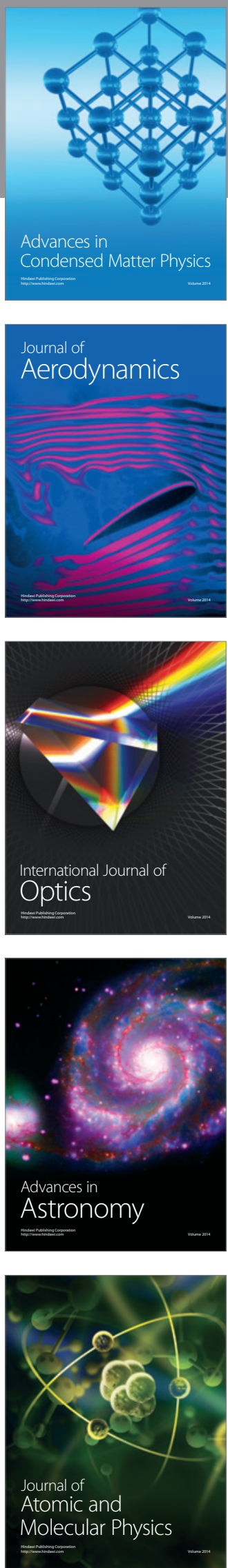